\title{
Transfer of encoding strategies in short-term memory
}

\author{
RONALD C. PETERSEN \\ Biomedical Laboratory, Building 3100, Edgewood Arsenal, Aberdeen Proving Ground, Maryland 21010 \\ and \\ ROBERT KARSH and RICHARD A. MONTY \\ United States Army Human Engineering Laboratory, Aberdeen Proving Ground, Maryland 21005
}

\begin{abstract}
The present experiment was designed to determine if a strategy imposed on a memory loaded task by a certain method of presentation of materials would transfer to a different display mode involving basically the same task but in a different format. It was found that, in general, spatial encoding strategies do transfer to different display formats requiring that the same task be performed.
\end{abstract}

The efficacy of a spatial encoding strategy in keepingtrack performance is well known (e.g., Monty, 1968; Munty. Taub. \& Laughery, 1965). This strategy in its most general form holds that subjects visualize a onedimensional array of the categories to be remembered and keep a mental tally of the number of occurrences of each category. Monty, Fisher, and Karsh (1967) have shown that materials which possess an inherent order, such as letters of the alphabet, lend themselves to this strategy more readily than those which are not ordered, such as geometric shapes. Furthermore, Monty (1968) demonstrated that performance can be enhanced by imposing an order on materials which do not possess an inherent order by presenting the categories to be tallied in constant positions on the display.

The present experiment was designed to assess the robustness of imposed strategies for keeping-track performance. A transfer design was used to determine if a strategy imposed by a certain method of presentation of the materials would transfer to a different display involving basically the same task but in a different format. In particular, the subjects were instructed to keep track of the number of occurrences of four categories of information. On pretransfer trials they were trained on une of three display formats: an ordered condition involving four information displays with each category always appearing on the same display, a scrambled condition involving four displays with each category appearing on any of the displays, and the single condition with the four categories appearing on only a single display.

Based on previous work by Monty and his associates (e.g., Monty, 1968; Monty, Fisher, \& Karsh, 1967), the

I his paper may be reproduced in full or in part for any purpose (if the Lnited States Government. Requests for reprints should be sent to Ronald C. Petersen. Biomedical Laboratory, Building 31(K). Edgewood Arsenal. Aberdeen Proving Ground. Maryland $21(1)$. subjects trained on the ordered display should outperform those trained on the single display on training because the ordered format facilitates adoption of the spatial encoding strategy. The single display, however, should lead to performance superior to that of the scrambled display because the latter interferes with the subjects' ability to adopt the spatial encoding strategy. Furthermore, on transfer trials when everyone is presented the four categories on a single display, if the ordered display has induced the subjects to adopt the spatial encoding strategy, then these subjects should perform at a level equal to or better than that of subjects trained on the single display. Alternatively, if the scrambled display has hindered acquisition of the spatial encoding strategy, these subjects should not perform as well as those trained on the single display.

\section{METHOD}

\section{Subjects}

Ninety-six students from Harford Community College, Churchville, Maryland served as paid volunteers.

\section{Apparatus and Procedures}

The apparatus and procedures have been described more fully elsewhere (Monty et al., 1965). Briefly, stimuli were projected one at a time onto the surface of rear projection display units. The subject's task was to observe and tally mentally each of four different stimuli (categories) presented sequentially and to record the tally in writing at the end of each sequence.

The subjects were randomly assigned to six groups of 16 each, which differed in terms of the stimulus class viewed (letters or symbols) and the method of presentation. Three groups viewed the letters $Q, R, S, T$, and three viewed the symbols "plus sign," "triangle," "square," and "heart." Two groups with each stimulus class first viewed the stimuli on a single display (single condition). Two groups viewed them on four adjacent displays, with each category always appearing on the same display (ordered condition). Letters were presented in the order (from left to right) $Q, R, S$, and T; symbols in the order "plus sign," "triangle," "square," and "heart." The remaining two groups also viewed the stimuli on four adjacent displays with each category appearing on all displays (scrambled condition). The position in 
ORDERED

SCRAMBLED

SINGLE

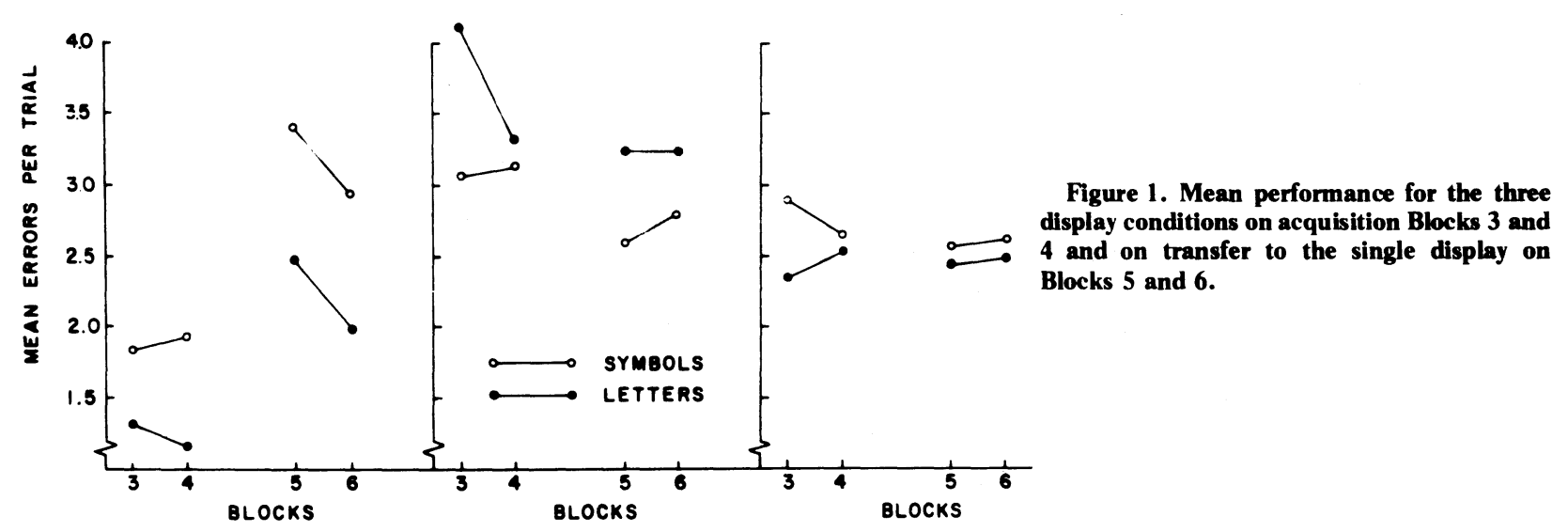

which each successive stimulus appeared was entirely random. In all cases, the actual sequence of categories presented to each group was identical, and the rate of presentation was held constant at one stimulus every $2 \mathrm{sec}$ with a $1-\mathrm{sec}$ stimulus on time and a $1-s e c$ interstimulus off time.

Trial length, defined as the total number of stimuli presented in a sequence, was included as a within-subjects variable. All subjects were presented with trial lengths of $8,12,16$ and 20 stimuli. Each of the four trial lengths was presented twice in random order during each of the four blocks of eight trials. A $2,500-\mathrm{Hz}$ tone signaled the beginning and end of each trial.

Following these first 32 trials, there was a brief pause of approximately $1 \mathrm{~min}$, and all subjects were given two additional blocks of eight trials but this time all subjects viewed all stimuli on a single display (single condition).

\section{RESULTS AND DISCUSSION}

The sum of the absolute difference between the number of stimuli presented in each category and the number reported in each category was used as the measure of performance. The absolute error scores for the two equal trial lengths within each block were summed and subjected to an analysis of variance (Butler, Kamlet, \& Monty, 1969) with stimulus class and presentation method as between effects and blocks and trial length as within effects. Since performance took two trial blocks to stabilize, only Blocks 3 and 4 were used in the analysis of pretransfer performance.

The data underlying the significant main effect for presentation method, $F(2,90)=10.64, p<.001$, and a subsequent Newman-Keuls test $(p<.05)$ indicated that the ordered condition was better than the single condition, which in turn surpassed the scrambled condition (mean errors per trial, respectively, of $1.56,2.61$, and 3.40). The main effect for trial length, $F(3,270)=102.90, p<.001$, also reached significance as did the Trial Length by Blocks interaction, $F(3,270)=6.75, p<.01$, simply indicating that the longer the trial length the poorer the performance but the greater the improvement across blocks. Of greater interest was the significant Presentation Method by Stimulus Class by Blocks interaction, $F(2,90)=3.70$, $p<.05$. The data underlying this interaction are shown in Figure 1. It can be seen that letters generally lead to better performance than symbols except for the scrambled condition where symbols unexpectedly led to better performance than letters. This latter finding is inconsistent with the work of Monty (1968), who found that letters always led to better performance than symbols. This discrepancy may be due to differences in on-off ratios in the two studies but should be the subject of further investigation. Finally, the Trial Length by Presentation Method interaction also reached significance, $F(6,270)=2.66, p<.05$, indicating that the longer the trial length the greater the differences between groups. In sum, from the results for the pretransfer trials, it appears that while method of presentation and the nature of the stimulus materials are both important, the former seems to predominate.

To assess performance on the transfer to a single display, difference scores obtained by subtracting performance on Block 4 from that on Block 5 were submitted to the same analysis of variance as that used for Blocks 3 and 4, exclusive of the Blocks factor. This analysis revealed a significant effect due to presentation method, $F(2,90)=19.88, p<.001$. A Newman-Keuls test indicated $(p<.05)$ that while performance improved to about the same extent for the single and scrambled conditions, it decreased for the ordered condition and was significantly different from the other two conditions. This finding implies that the relatively good performance for the ordered groups on pretransfer trials was affected by transfer to a single display to a greater extent than was performance in the other two conditions.

Finally, to assess post-transfer performance, the data for Blocks 5 and 6 were subjected to the same analysis of variance as that used for Blocks 3 and 4. Only the main effect for trial length, $F(3,270)=58.30, p<.001$, and the Trial Length by Blocks interaction, $F(3,270)=3.57, \quad p<.05$, reached significance. The interaction indicated that subjects improved to a greater 
extent from Block 5 to Block 6 on the shorter trial length than on the longer. Of primary importance in this analysis was the lack of a significant effect due to presentation method on the post-transfer trials. In spite of differences on the pretransfer trials, the groups converged when transferred to the single display.

The results indicate that while the ordered condition was most dramatically affected by transfer to a single display, these subjects still performed as well as those who were trained on the single display. Their performance was relatively error free on pretransfer trials, largely due to the ordered display format, and, therefore, could not be expected to improve on transfer to a more difficult format. Despite this, their performance on transfer was no different from that of the subjects who were trained on the transfer task, indicating that they did benefit from training on the ordered display.

The scrambled condition subjects, on the other hand, were expected to perform more poorly than the single condition subjects on transfer because of their relatively poor performance on acquisition. As post-transfer performance indicated, however, while they made more errors than the subjects in the single condition, the difference was not significant. In sum, it appears that while the presentation methods led to disparate performance on acquisition, the subjects in the ordered and scrambled conditions abstracted enough information regarding keeping track in general to facilitate their performance on transfer. It can be concluded, therefore, that general strategies regarding keeping-track performance do transfer across display formats.

\section{REFERENCES}

Butler, D. H., Kamlet, A. S., \& Monty, R. A. A multi-purpose analysis of variance FORTRAN IV computer program. Psychonomic Monograph Supplement. 1969, 2. 301-319 (Whole No. 32).

Monty. R. A. Spatial encoding strategies in sequential short-term memory. Journal of Experimental Psychology. 1968. 77, 506-508.

Monty. R. A.. Fisher, D. F.. \& Karsh, R. Stimulus characteristics and spatial encoding in sequential short-term memory. Journal of Psychology, 1967, 65, 109-116.

Monty. R. A.. Taub. H. A., \& Laughery, K. R. Keeping track of sequential events: Effects of rate. categories and trial length. Journal of Experimental Psychology, 1965, 69, 224-229.

(Received for publication January 16, 1976.) 